\title{
A Simple Algorithm to Strengthen the Brightness of Color Images
}

\author{
Hua ZHANG \\ Department of Computer and Communication, Weifang University, Weifang261061,China
}

\begin{abstract}
This paper puts forward a simple algorithm to make color images lighter based on Wavelet transformation and histogram smoothing. This algorithm uses HIS space which is based on human visual features. Without changing hue, it can conduct histogram smoothing in low brightness frequency domain through wavelet decomposition and make the images lighter, which at the same time avoids vagueness of details and increase of noises. According to experiment results, this algorithm can make low brightness color images lighter effectively with low time complexity.
\end{abstract}

Index Terms: HIS color space, saturation ratio, brightness, Wavelet transformation, histogram smoothing

(C) 2011 Published by MECS Publisher. Selection and/or peer review under responsibility of the Research Association of Modern Education and Computer Science

\section{Introduction}

Humans convey 70 percent of their information through visible sensation. Thus images are an important medium and means for conveying information. Because of different kinds of reasons, damages or noises can be added into images during transmission and equipment read process which makes the images difficult to recognize. This explains why image enhancement processing has been paid more and more attention. There has been many mature and efficient ways for enhancement processing of grey images while the enhancement processing of color images has not attract enough attention. Compared with grey images, color images contain richer information, have stronger equipment processing capacity and can be applied to wider areas. In the processing of images, the use of colors is very important. There are two reasons [1]: firstly, in automatic image analysis, color is a useful descriptive tool which can make it simpler to recognize and abstract an object from a scene; secondly, when analyzing images, humans can identify about only 20 grades of gray, but hundreds and thousands of colors. In order to strengthen color images, two problems [2] should be solved: firstly, the way to keep the hue unchanged; secondly, the way to strengthen the image with hue unchanged.

In order to solve these two problems, this paper puts forward a new algorithm to strengthen color images which can at the same time erase noises and strengthen the brightness and saturation ratio of the image.

* Corresponding author.

E-mail address: wfujsj@163.com 


\section{The Algorithm to Strengthen Color Images}

\subsection{HIS Space}

HIS space was established from human's psychological perception. $\mathrm{H}$ (hue) means the dominant wave length of a color in color sped rum; S (saturation ratio) means the degree of purity of a color; I (brightness) is the equilibrium quantity of sense. Brightness weight and hue weight are separated and are irrelevant to color information of images. $\mathrm{H}$ and $\mathrm{S}$ weight are highly relevant to the way that humans sense colors. The ability of $\mathrm{H}$ weight to describe colors is relatively the closest to humans' visible sensation and thus has stronger abilities[3]. The above features make HIS color space very proper for the processing of color images.

\subsection{The Enhancement Processing of Brightness Sections}

\section{1) Wavelet decomposition}

There are mainly two enhancement ways: airspace enhancement method and wave filter enhancement method which can be improved and used or directly used in the enhancement of images [4-7]. airspace enhancement method can make the whole image pixel even and better for further processing. Histogram smoothing is an effective airspace enhancement method. However, as the image details and noises mainly exist in the highfrequency sections of the image, this algorithm will lead to the lose of image details through its unification of low-frequency gray scale. It enhanced noises while enhancement the image. Wavelet analysis is a time frequency localization analysis method with changeable shape, time window and frequency, and unchangeable window size. It has good localization feature in time domain and frequency domain. After being separated by wavelet, the image will be divided into four sub-bands: LI, LH, HL and HH. LL reflects the low-frequency information in horizontal and vertical directions. HH reflects the high-frequency information in horizontal and vertical directions. According to the above analysis, we can use histogram smoothing method only in lowfrequency sections, which can strengthen the image as well as avoiding the vagueness of details and the increase of noises.

The first grade decomposition of image $\mathrm{H}$ with wavelet base "sym 4" is shown in Fig. 1.

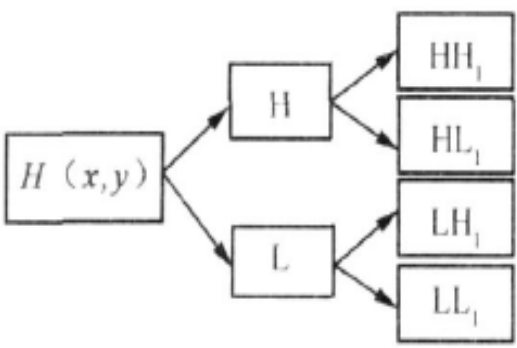

Fig 1. First grade wavelet decomposition of the image 


\section{2) Histogram smoothing}

If the target grade of gray of two objects close to each other differs by less than 10, human eyes will not feel the difference, nor can they distinguish the two objects. The histogram smoothing with enhanced spatial domain will strengthen the contrast of the image through redistributing the grades of gray evenly.

According to the above analysis, if the grade of gray limit of the brightness image range from 0 to 255 , we choose sub-band $L L_{1}$ to conduct histogram smoothing. If the frequency of $i$ gray grade $r_{i}$ is $n_{i}$, its probability value is $p_{r}\left(r_{i}\right)=n_{r} / n$ (" $n$ " is the total number of pixel) and $r_{i}$ satisfies normalization condition. The function expression of image histogram smoothing is as follows:

$$
s_{i}=T\left(r_{i}\right)=\sum_{i=0}^{k-1} p_{r}\left(r_{i}\right)=\sum_{i=0}^{k-1} n_{i} / n
$$

In this expression, " $k$ ” means grades of gray.

As the physical meaning of histogram is the total number of pixel contained in some grade of gray "r", a certain part of histogram shows that the total number of pixel contained in this part of grades of gray limit equals that of a new part of grade of gray " $\mathrm{s}$ " which is reflected one by one through monotonic increasing function. Thus we have the following expression:

$$
p_{s}(s) \mathrm{d} s=p_{r}(r) \mathrm{d} r
$$

If we calculate the derivative of "s" from the two sides of the expression, we get:

$$
\left.p_{s}(s)=\frac{\mathrm{d}}{\mathrm{d} d}\left[\int_{-\mathrm{J}}^{r} p_{r}(r) \mathrm{d} r\right]=p_{r} \frac{\mathrm{d} r}{\mathrm{~d} s} T^{-1}(s)\right]
$$

The smoothing histogram demands $p_{s}(s)$ to be constant. Suppose $p_{s}(s)=1$, we can get from the above two expressions:

$$
\mathrm{d} s=p_{r}(r) \mathrm{d} r
$$

Calculate the integral from the two sides, we get:

$$
s=T(r)={ }_{0}^{r} p_{r}(r) \mathrm{d} r
$$

At the right side of this expression is the cumulative distribution function of $\mathrm{p}_{\mathrm{r}}(\mathrm{r})$. It reflects that histogram smoothing could be achieved when transformation function is the cumulative distribution function of " $\mathrm{r}$ ", which is shown in Fig. 2. 


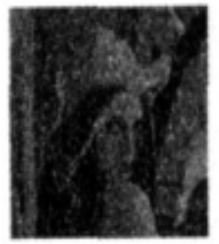

a)

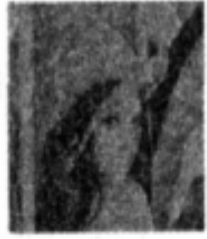

b)

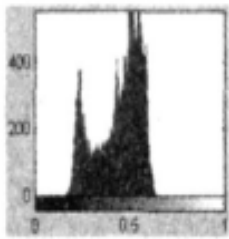

c)

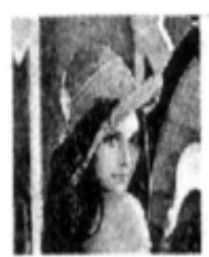

c)

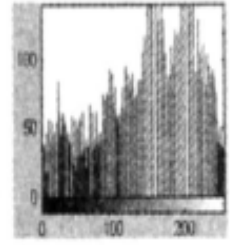

d)

Fig 2. The comparison of the results before and after histogram smoothing. a) Original image; b) the brightness LL1 weight after wavelet decomposition; c) the histogram of image b); d) the smoothing results of image b); e) the histogram of image d)

\section{3) Wavelet reconstruction}

Suppose after histogram smoothing, LL becomes image $\mathrm{LL}_{1}$. Conduct wavelet reconstruction with $\mathrm{LL}_{1}$ and LH,HH,HL.

\subsection{Saturation Ratio Enhancement}

In order to ensure that after enhancement, the image will become brighter in color, we adapted the non linearity index of saturation ratio weight to increase the dynamic range of the change of colors and increase its contrast. Index tensile $S^{\prime}=S^{\text {a }} \quad$ (“a” means tensile determiner and decides the degree of saturation ratio weight).

\subsection{Normalization}

H, S, I weight normalization into RGB space.

\section{Results Analysis}

In Fig. 3, a) is the low light level original image.

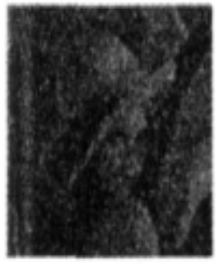

a)

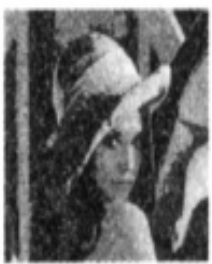

b)

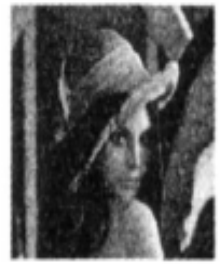

c)

Fig 3. Results analysis. a) The original image; b) Traditional algorithm enhancement effect; c) The algorithm result of this paper 
After histogram smoothing I space into RGB space, we get image b). Image c) is the result of the algorithm put forward by this paper. In order to check the effectiveness of this algorithm, we calculated the average value and variance of the image and invesgated image a) through the method of measuring brightness and contrast provided in Jobson, Ralanan and Woodell's book(2002).

Table 1 Comment on the enhancement results

\begin{tabular}{|l|l|l|l|}
\hline Comment Parameters & Original Image & Traditional histogram balanced algorithm & The algorithm in this paper \\
\hline Image average value & 85.8092 & 149.3367 & 99.5776 \\
\hline Image variance & 2097.4 & 5579.7 & 6368.1 \\
\hline C & & 0.7403 & 0.8605 \\
\hline L & & 1.6602 & 2.0361 \\
\hline
\end{tabular}

\section{Conclusion}

This thesis puts forward a color images enhancement algorithm with unchanged hue based on HIS color space by combining the advantages of wavelet transformation and histogram balanced. It aims at solving the problem that the traditional color images enhancement algorithm may easily lose some details in gray image space. This new algorithm conducts histogram smoothing in low-brightness domain to strengthen the brightness sections of the image through wavelet decomposition. It reconstructs the enhanced "I" image. It uses index tensile algorithm in saturation ratio space and enhances saturation ratio. This algorithm is simple to calculate and effective in enhancement the images, especially low brightness images.

\section{References}

[1] Qiuqi Ruan. Processing of Digital Images[M].Beijing: Publishing House of Electronics Industry, 2001.(in Chinese)

[2] Ping Wang, Hao Cheng, Yingxi Luo. Color Image Enhancement Based on Hue Invariability [J]. Journal of Image and Graphics, 2007,12(7): 1173. (in Chinese)

[3] Xiaowei Han. Study of the Key Technologies in Color Image Processing[D].ShenYang: Northeastern University, 2005. (in Chinese)

[4] Hong L,wan Y F, Jain A..Fingeip rint image enhancement algorithm and performance evaluation[J]. IEEE Trans on Pattern Analysis and Machine lntelligenc;1998,20(8): 777.

[5] Willis A J, M yers L A costeffective fingerp rint recognition system for use with low quality prints and damaged fingertips[J]. Pattern Recognition, 2001, 34(2): 255.

[6] Sherlock B G, M onro D M, M illaml K.. Fingerp rint enhancement by directional Fourier filter[ J]. IEEE Proceedings of Vision Inage and Signal Processing 1994,141(2): 87.

[7] Yanhong Zhang, Dewen Hou.The Image Enhancement Algorithm Based on Wavelet Frequency Sharing and One-time Balancing[J]Computer Application and Software, 2007,24(11): 159. (in Chinese)

[8] Jobson DJ, RalananZU, WoodellGA.The statistics of visual representation[ C]//Processing of SPIE Visual 1nfoanation processing Xl Washington SPIE Press 2002 25-35. 